\section{Microbiological water quality monitoring in a resource-limited urban area: a study in Cameroon, Africa}

\author{
Andrew W. Nelson, 1,2,4 \\ Leah M. Feazel, 1,3,4,5 \\ Charles E. Robertson, ${ }^{2}$ John R. Spear, ${ }^{1}$ \\ Daniel N. Frank $k^{3,5}$ \\ 1Department of Civil and Environmental \\ Engineering, Colorado School of Mines, \\ Golden; ${ }^{2}$ Molecular, Cellular, \\ Developmental Biology, University of \\ Colorado, Boulder; ${ }^{3}$ Division of Infectious \\ Diseases, School of Medicine, University \\ of Colorado Anschutz Medical Campus, \\ Aurora; ${ }^{4}$ DOORs Cameroon Inc., Boulder; \\ ${ }^{5}$ Microbiome Research Consortium, \\ University of Colorado Anschutz Medical \\ Campus, Aurora, CO, USA
}

\section{Abstract}

In resource-limited developing nations, such as Cameroon, the expense of modern water-quality monitoring techniques is prohibitive to frequent water testing, as is done in the developed world. Inexpensive, shelf-stable 3M ${ }^{\mathrm{TM}}$ Petrifilm ${ }^{\mathrm{TM}}$ Escherichia coli/Coliform Count Plates potentially can provide significant opportunity for routine water-quality monitoring in the absence of infrastructure for state-of-the-art testing. We used shelf-stable $E$. coli/coliform culture plates to assess the water quality at twenty sampling sites in Kumbo, Cameroon. Culture results from treated and untreated sources were compared to modern bacterial DNA pyrosequencing methods using established bioinformatics and statistical tools. Petrifilms were reproducible between replicates and sampling dates. Additionally, cultivation on Petrifilms suggests that treatment by the Kumbo Water Authority (KWA) greatly improves water quality as compared with untreated river and rainwater. The majority of sequences detected were representative of common water and soil microbes, with a minority of sequences $(<40 \%)$ identified as belonging to genera common in fecal matter and/or causes of human disease. Water sources had variable DNA sequence counts that correlated significantly with the culture count data and may therefore be a proxy for bacterial load. Although the KWA does not meet Western standards for water quality (less than one coliform per $100 \mathrm{~mL}$ ), KWA piped water is safer than locally available alternative water sources such as river and rainwater. The culture-based technology described is easily transferrable to resource-limited areas and provides local water authorities with valuable microbiological safety information with potential to protect public health in developing nations.

\section{Introduction}

In 2004, the World Health Organization (WHO) estimated that 2.2 million people die annually from diarrheal diseases, representing $3.7 \%$ of global mortality. ${ }^{1}$ Most of these deaths are due to preventable waterborne illnesses that occur in developing nations because of inadequate sanitation. Consequently, developed countries such as the United States and Canada sponsor improved sanitation water projects that are designed to lessen the risk of waterborne illnesses. However, one weakness of such water projects is the limited financial and technical involvement of developed nations after water projects are completed.

As privatization replaces government subsidization, water authorities in developing countries often must shift to a user fee-based system. Billing residents for purified water presents a challenge because many citizens are either unable or unwilling to pay for public services such as improved drinking water. ${ }^{2}$ Furthermore, booming populations and urban influx place greater strain on existing infrastructure, explaining in part why the proportion of Africans with access to clean water has not increased since $1990 .^{3}$ Therefore, water authorities typically must operate on highly conservative budgets unless supported by outside funding. Low operating budgets often result in compromised water treatment and reduced ancillary services such as water quality monitoring. Thus, the efficacy and sustainability of improved public water works in developing nations is questionable.

In this study we evaluated the efficacy of a low-cost, technically simple bacteriological water quality test, with the hope of facilitating more frequent monitoring in a resource-limited area. The site of this study was Kumbo, Cameroon, a small city in Sub-Saharan West Africa (Figure 1). In 1968, with a population of less than 13,000 , the city received assistance from the Canadian government to construct a drinking water treatment facility. ${ }^{4,5}$ Since completion in 1970, the operating entity has changed several times, but today the Kumbo Water Authority (KWA) manages the treatment facility with support from the Kumbo City Council. Although the distribution network has greatly expanded in recent years (currently delivering $>280,000 \mathrm{~m}^{3}$ per year), the treatment facility has experienced few upgrades since 1970 . Now, with more than 100,000 residents, the KWA cannot meet the demand.
Correspondence: Daniel N. Frank, Division of Infectious Diseases, School of Medicine and Microbiome Research Consortium, University of Colorado Anschutz Medical Campus, 12700 E. 19th Ave, MS B168 Bldg P15, Aurora, C0 USA 80045.

Tel: + 1.303.724.5536 - Fax: +1.303.724.4926.

E-mail: daniel.frank@ucdenver.edu

Key words: coliform, development, microbiology, pyrosequencing, water.

Acknowledgments: we thank Jackson Lee of the Colorado School of Mines, as well as Dr. Norman Pace and Kimberly Ross of the University of Colorado-Boulder for their contributions of ideas and materials. Additionally, we thank Sika Harris Bronson for his assistance with sample collection and all employees of the KumboWater Authority and Kumbo City Council for their contributions and assistance. This project was funded in part by grants to AWN and LMF from the Pink Foundation, through the Office of International Programs at the Colorado School of Mines. DNF and CER were supported by NIH grant HG005964.

Contributions: AWN, LMF, contributed equally; AWN, LMF, DNF, JRS protocol conception and design; AWN, LMF data collection; AWN, LMF, CER, DNF data analysis; AWN, LMF, manuscript preparation; AWN, LMF, DNF, CER, JRS drafting and editing. All authors gave final approval.

Conflict of interests: the authors report no conflict of interests.

Received for publication: 15 November 2011.

Revision received: 9 March 2012.

Accepted for publication: 3 August 2012.

This work is licensed under a Creative Commons Attribution NonCommercial 3.0 License (CC BYNC 3.0).

(C) Copyright A.W. Nelson et al., 2012

Licensee PAGEPress, Italy

Journal of Public Health in Africa 2012; 3:e19 doi:10.4081/jphia.2012.e19

Water shortages are not uncommon. ${ }^{6}$ Furthermore, the original asbestos cement water mains are deteriorating and must be replaced with relatively expensive PVC pipes. Due to salaries, maintenance and chemical costs, the KWA can only afford to monitor biannually for ions, heavy metals, and microbiological agents, at a cost of approximately 600 USD per sampling event. ${ }^{7}$ Water samples are shipped to the nearest equipped laboratory in Mutangene, Cameroon at a distance of $400 \mathrm{~km}$ ( $8+\mathrm{h}$ by car). Furthermore, the KWA has little control over how the samples are handled in transit or how quickly the results are processed. At a sampling frequency of twice per year, the city cannot make informed decisions on the safety of supplied water. In a 
developed nation, such as the United States of America, a city of an equivalent size to Kumbo must sample a minimum of 100 times per month for microbial safety. ${ }^{8}$ With more frequent monitoring, Kumbo could reduce waterborne illness by issuing boil water notices, as Boston, USA issued to protect two million residents in May $2010 .^{9}$

Water authorities in Kumbohave demonstrated interest in monitoring (Njoka Shiyntum, personal communication, 2010). However, conventional monitoring technologies recommended by the WHO are too expensive given the annual budget of the KWA. ${ }^{7}$ The WHO recommends monitoring fecal coliforms as an indicator for biological water quality using the most probable number technique and filter-based techniques coupled with media specific for lactase and $\beta$-D-glucuronidase activity. ${ }^{10}$ Fecal coliforms, such as Escherichia coli, are thermotolerant bacteria that are associated with human and animal fecal waste and are characterized by lactase and $\beta$-D-glucuronidase activities. Coliforms have been used as indicators of water purity since the early 1900 s, but their presence is not necessarily due to fecal contamination and does not correlate well with protozoan parasites or viruses. ${ }^{11}$ However, in the absence of modern equipment for the detection and removal of such organisms, the detection of nominal $E$. coli and coliforms as indicator organisms provides water authorities an efficient and simple method to monitor contamination events.

Microbiological water quality monitoring is easily and routinely performed in developed nations. However, standard techniques require relatively expensive laboratory equipment such as autoclaves, petri dishes, perishable growth media, and filtration devices. New technologies for quick coliform identification exist, such as $3 \mathrm{M}^{\mathrm{TM}}$ Petrifilm $^{\mathrm{TM}} E$. coli/Coliform Count Plates (hereafter referred to simply as Petrifilms). In this study, we sought to (i) assess the utility of Petrifilms for routine water quality monitoring in an urban resource-limited area and (ii) test the current efficacy of water treatment in Kumbo through use of Petrifilms and modern DNA sequencing methods.

Several groups have used Petrifilms as fecal coliform count plates in the developing world, primarily in rural regions. ${ }^{12,13}$ We chose to study Petrifilms in an urban setting, specifically to determine their applicability in frequent water quality monitoring. Petrifilms provide easily interpretable, colorimetric phenotype information and bypass many conventional monitoring expenses, requiring only an incubator. Additionally, these dehydrated, shelf-stable fecal coliform culture plates are inexpensive ( $<1$ USD per test), extremely lightweight and compact, which makes them easy to transport. The plates detect acid formation, lactose- fermentation, and have an indicator dye for $\beta$ D-glucuronidase activity, the latter of which differentiates nominal $E$. coli from other coliforms. In contrast to broth culture approaches, plate-based assays can detect and enumerate polymicrobial contaminants in water samples.

\section{Materials and Methods}

\section{Study area and collaborations}

The study site was Kumbo, Bui Division, Northwest Region, Cameroon, Africa $\left(6^{\circ} 1218 \mathrm{~N}, 10^{\circ} 416 \mathrm{E}\right)$. This project was planned and executed with the support and permission of Kumbo City Mayor Njong Fonyuy Donatus, the Kumbo City Council, and the KWA. A water technician from KWA assisted in all site access and sample collection, and was trained to use Petrifilms.

\section{Sample sites and water collection}

We sampled both treated and untreated water sources on two separate occasions, approximately one month apart, in June and July of 2010. During each sampling date, ten water samples were collected for a total of twenty. Samples were collected from the KWA facility, its distribution network, and alternative untreated drinking water sources. Specifically, we collected samples on two occasions from the raw source water supplying KWA, the outflow of the sand filtration system, and a tap directly linked to the chlorinated water holding tank. Within the KWA distribution network, we sam- pled four indoor taps and seven outdoor taps throughout Kumbo. Additionally, we collected three samples from alternative, untreated drinking water sources, namely rain collection buckets and river water (River Bui). Surface water and water from rain buckets were collected directly into clean, sterile $0.5 \mathrm{~L}$ Nalgene ${ }^{\circledR}$ bottles. All taps were flushed for $60 \mathrm{~s}$ to remove biofilms and stagnant water before collection into sterile $0.5 \mathrm{~L}$ Nalgene ${ }^{\circledR}$ bottles.

\section{Sample processing}

Collected water samples were divided and either filtered for DNA extraction or plated in duplicate for culture within $1 \mathrm{~h}$ of sample collection. Water (70-150 mL) was filtered through a $0.2 \mathrm{~m}$ polycarbonate filter (Isopore $^{\mathrm{TM}}$ Membrane Filters, Millipore Corp., MA, USA) using a hand vacuum pump. The filters were stored in $2 \mathrm{~mL}$ of sterile $95 \%$ ethanol until DNA extraction. For each site, two 3M $\mathrm{M}^{\mathrm{TM}}$ Petrifilm $^{\mathrm{TM}}$ E. coli/Coliform Count Plates (3M, St. Paul, MN, USA) were plated. One milliliter $(1 \mathrm{~mL})$ of water was poured onto the plates and the plates were incubated for $24 \mathrm{~h}$ at $>37^{\circ} \mathrm{C}$, as per the manufacturer's recommendation and previously described. ${ }^{14}$

\section{Colony counts}

Colonies were counted according to the plate manufacturer's instructions and categorized as follows: blue with gas, red with gas, or red without gas. Red non-gas-producing colonies are thermotolerant bacteria with little or no lactase activity. Red gas-producing

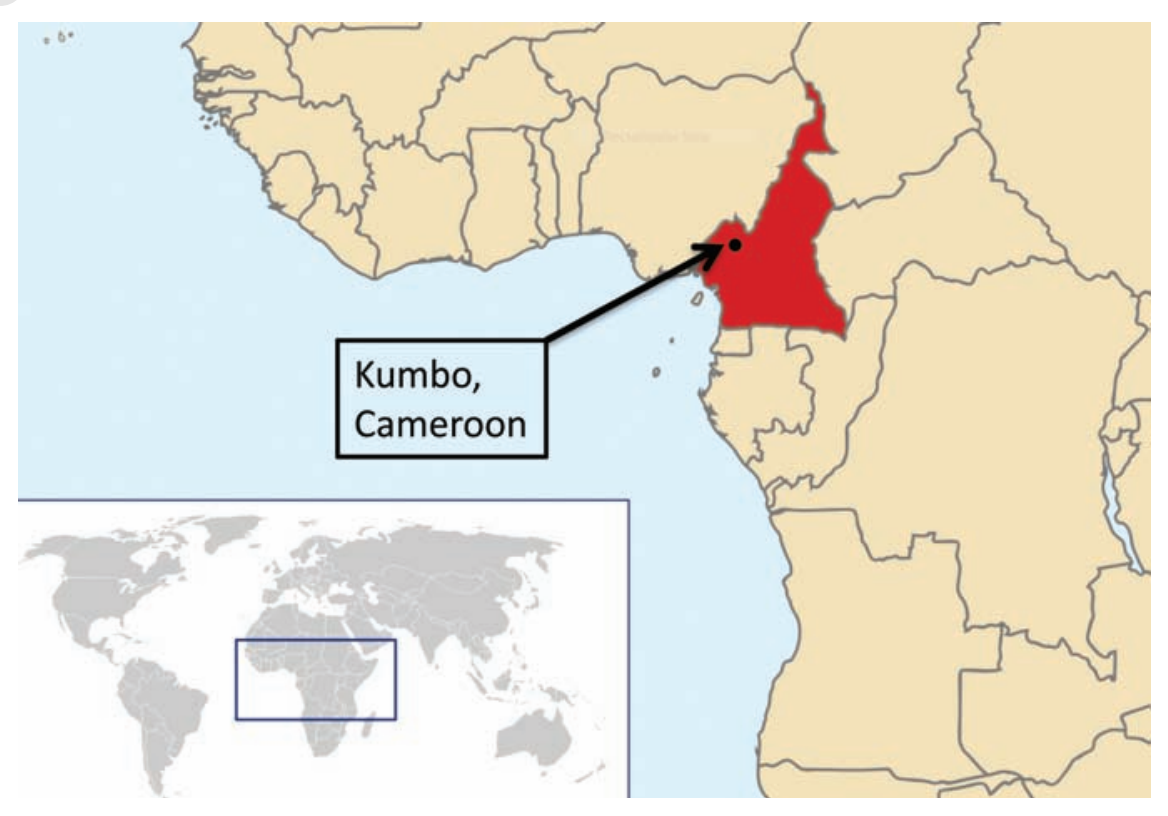

Figure 1. Study site location. 
colonies are thermotolerant bacteria that have lactase activity, and are therefore likely coliforms. Blue colonies are thermotolerant and have lactase and $\beta$-D-glucuronidase activity, a phenotype consistent with $E$. coli. Hereafter all thermotolerant bacteria are referred to as TTB, lactose fermenting bacteria as LFB, and colonies with $\beta$-D-glucuronidase activity as BDGB. Photographs were taken of each Petrifilm with a digital camera (Samsung S630), and manual colony counts were confirmed by computer-assisted enumeration using the software package IMAGEJ (version $1.44 \mathrm{p})$ with Cell Counter Plugin (http://rsb.info.nih.gov/ij/).

\section{DNA extraction}

Prior to DNA extraction, filters were dried by lyophilization of the storage ethanol. Then, one-half of each filter was extracted using the MoBio UltraClean Fecal DNA Kit, following the manufacturer's protocol (MoBio Laboratories Inc, Carlsbad, CA, USA) ${ }^{15}$. All DNA extraction and polymerase chain reaction (PCR) steps were performed in a UV light-decontaminated laminar flow hood.

\section{Multiplexed pyrosequencing}

Thirty $\mu \mathrm{L}$ PCR reactions contained 1x Nova Taq Mastermix (Merck KGaA, Darmstadt, Germany), ${ }^{16} 0.4 \mu \mathrm{M}$ primer $27 \mathrm{~F}-\mathrm{YM}+3,{ }^{17} 0.4$ $\mu \mathrm{M}$ barcoded primer $534 \mathrm{R}, 18,19$ and $2 \mu \mathrm{L}$ mixed community DNA. Each sample was assigned to three unique barcodes for pyrosequencing. The PCR protocol was as recommended by the manufacturer, with a $52^{\circ} \mathrm{C}$ annealing temperature and a total of 39 cycles. PCR product concentrationswere normalized using a SequalPrep $^{\mathrm{TM}}$ Normalization Plate Kit (Invitrogen Inc., CA, USA), following the manufacturer's protocol. The eluted PCR products were pooled and lyophilized to $35 \mu \mathrm{L}$ total volume. The pool was divided in two and both halves electrophoresed through a $1 \%$ agarose gel in Tris/Acetate/EDTA and product excised with a sterile razor blade under low-wavelength ultraviolet light. DNA was eluted from the gel slice using the Montage ${ }^{\mathrm{TM}}$ DNA Gel Extraction Kit (Millipore Corp., MA, USA). The gel eluates were re-combined and lyophilized to a final volume of $30 \mu \mathrm{L}$. Half of the final pooled DNA was provided to the Colorado Consortium for Comparative Genomics for pyrosequencing on a 454/Roche Life Sciences GS-FLX instrument using titanium chemistry (Roche Life Sciences, Indianapolis, IN, USA).

\section{Sequence analysis}

A total of 32,425 high-quality pyrosequences from 19 samples (one rainwater sample had too little volume to filter) were obtained. The software used for sequence analysis consisted of the function specific tools that were consid- ered to be the standards in the academic community at the onset of this project. Pyrosequences were sorted into libraries by barcode using bartab. ${ }^{19}$ All pyrosequences were screened for nucleotide quality (bases at 5 ' and 3' ends with mean $Q<20$ over a 10 n.t. window were discarded), ambiguous bases (sequences with $>1 \mathrm{~N}$ were discarded), and minimum length (sequences $<150$ n.t. were discarded). The Infernal RNA alignment too ${ }^{20}$ was used to screen all sequences for fidelity to a covariance model (CM) derived from bacterial small subunit rRNA secondary structure models provided by the lab of Dr. Robin Gutell. ${ }^{21,22}$ Sequences with poor fidelity to the bacterial CM model were removed from all subsequent analyses. Chimera screening was performed by the tool ChimeraSlayer, which required sequence alignment with $N A S T-i E r^{23}$ Putative chimeras and other sequences that could not be aligned by NAST-iEr were removed from subsequent analyses. Genus-level taxonomic assignments were produced by the $R D P$ Classifier, which performs naïve Bayesian taxonomic classification versus a training set. ${ }^{24}$ Bioinformatic analyses, including calculation of ecological indices of richness (e.g., $\mathrm{S}_{\mathrm{obs}}$, $\mathrm{S}_{\text {chao }}$ ), diversity (e.g., Shannon's index), and

sampling coverage (e.g., Good's index), were conducted with the software tool biodiv embedded within the sequence analysis pipeline. ${ }^{25}$ Microsoft Excel was used for Student's T-tests following logit transformation of percent abundance data and Anscombe transformation of count data.

\section{Results}

Water passing through the KWA treatment facility became significantly less contaminated by heterotrophic bacteria (Figure 2A). Raw water entering the facility contained a mean of $74.3 \pm 39.8$ TTB, $25.8 \pm 14.2 \mathrm{LFB}$, and $2.5 \pm 1.9$ BDGB. Water from the sand filtration bed contained $48.3 \pm 9.0$ TTB, $15.8 \pm 5.3 \mathrm{LFB}$, and $3.3 \pm 2.2$ BDGB. Chlorination reduced the bacterial loads in the holding tank to only 1 colony of TTB in four replicates, and no LFB or BDGB were detected. Samples collected from taps in the distribution network contained higher bacterial loads than water from holding tank. Specifically, indoor taps contained $8.0 \pm 12.0$ TTB, $0.5 \pm 0.9$ LFB, with no detectable BDGB and outdoor taps contained 1.6 \pm 1.6 TTB,
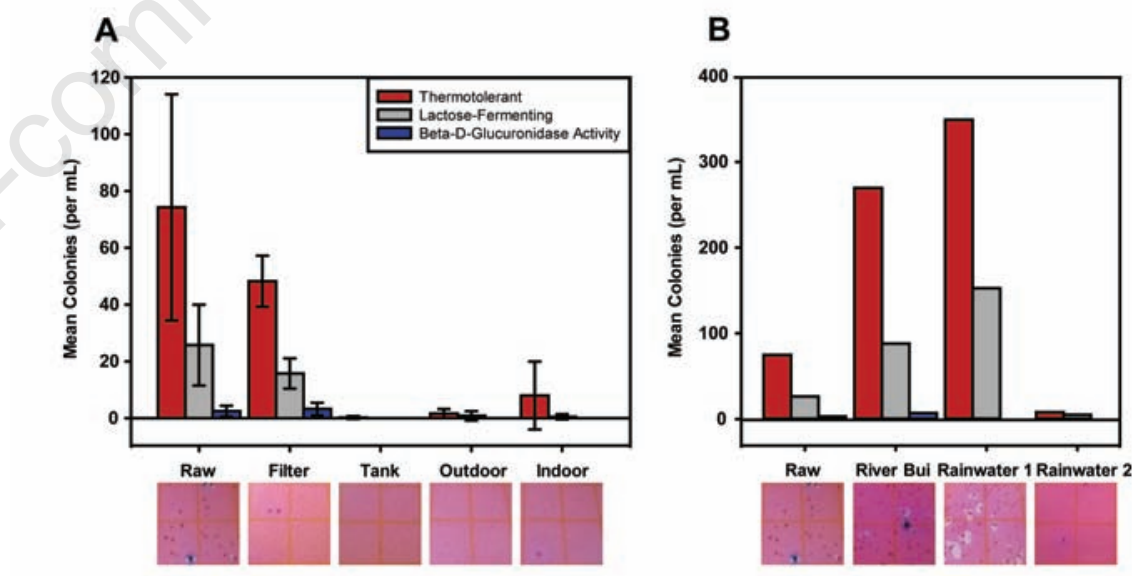

Figure 2. Results of cultivation with $4-\mathrm{cm}^{2}$ representative Petrifilm image. (A) Mean thermotolerant, lactose fermenting and $\beta$-D-glucuronidase active colony counts are displayed with standard deviations for sample sites within the Kumbo Water Authority (KWA) distribution system (Raw=untreated water source for KWA, Filter=rapid sand filtered water, tank=filtered and chlorinated water covered holding tank, outdoor=select outdoor water faucets serviced by KWA, indoor=select indoor water faucets serviced by KWA). Each sample was plated in duplicate. Raw, Filter and Tank had samples from two time points, Outdoor had seven and Indoor had four samples, respectively, collected over two sampling dates. (B) Mean thermotolerant, lactose fermenting and $\beta$-D-glucuronidase active colony counts are displayed for alternative water sources in Kumbo. Rainwater 1 represents water collected from an uncleaned bucket. Rainwater 2 represents water collected from the same bucket after scrubbing with bleach solution. River Bui, Rainwater 1, and Rainwater 2 have one sample each, plated in duplicate. 
$0.9 \pm 1.7$ LFB, again with no detectable BDGB.

A wide range of colony counts was observed in untreated water sources (Figure 2B), which included the raw water input into the KWA (shown also in Figure 2A), surface water from River Bui, and rainwater from a collection bucket. One rainwater sample (Rainwater 1) was collected from the eaves of a roof into an untreated bucket, whereas a second sample (Rainwater 2) was collected from the same bucket after it was sanitized with bleach and protected from roof runoff. Water from River Bui contained a mean bacterial load of 269 TTB, 87.5 LFB, and 6.5 BDGB (Figure 2B). Rainwater 1 had the highest measured bacterial load of all samples with 348.5 TTB, 152 LFB, and no detectable BDGB. After water safety precautions, Rainwater 2 had 7.5 TTB, 4.5 LFB, and no detectable BDGB.

In order to further analyze the microbiology of the Kumbo water treatment and distribution system, filtered water samples were evaluated by culture-independent analysis of $16 \mathrm{~S}$ rRNA gene sequences by broad-range PCR and pyrosequencing. Water sources had variable sequence counts, (mean 762-3, 210 sequences per sample type) which may be a rough proxy for the bacterial load, as these values correlate significantly with the culture count data $\left(\mathrm{P}=9.9 \times 10^{-6}\right.$; Supplemental Table 1$)$. As expected, the majority of sequences detected were representative of common water and soil microbes, with a minority of sequences $(<40 \%)$ identified as belonging to genera common in fecal matter and/or causes of human disease (Figure 3; The complete annotated sequence data for all individual sites is available in Supplemental Table 2). Because the latter microorganisms are of interest for waterquality monitoring, our analysis focused primarily on these relatively rarer sequences.

Both aerobic and anaerobic bacterial groups were detected by sequencing. The dominant sequence from an aerobic bacterial group of potential human-health interest belonged to the genus Bacillus ( $>9 \%$ of total sequences), which is common in the environment and generally nonpathogenic. This genus was identified in all treated and untreated water types. Sequences representative of another abundant genus, Staphylococcus (>8\%), were particularly abundant in tap water samples (1 of 4 indoor and 2 of 7 outdoor). When present, Staphylococcus generally comprised a high percentage of total sequences from those sites (5.5\%-31\%; Supplemental Table 2), possibly due to surface contamination of the taps from multiple users, and/or biofilm growth in the local tap lines. The Escherichia/Shigellagenus was extremely rare in the sequence data, comprising only $0.34 \%$ of total sequences, with the majority from the sand filter samples. Many of the anaerobes detected by sequence belong to non-pathogenic genera (e.g. Bifidobacterium spp.), the dominant anaer- obe sequences belonged to the genus Clostridium, which includes several water-borne fecal microorganisms such as $C$. difficile and $C$. perfringens. ${ }^{26}$ Untreated waters had the highest abundance of Clostridium spp. sequences (2.2$15 \%$ ), with lower abundance in samples from the KWA treatment tank and city taps (0.09$1.3 \%)$. The most prevalent genus identified by sequence that is commonly associated with feces was an uncultured group of organisms known as TM7 (5.7\% of total sequences), which has been identified in a range of aerobic and anaerobic specimens including human feces, sub-gingival crevices, and surface waters. ${ }^{27}$ TM7 sequences were particularly abundant in samples with higher fecal contamination as detected by culture, possibly indicating that TM7 DNA could be used to detect contaminated water.

\section{Discussion}

Petrifilm plates provide easily interpretable, colorimetric phenotypic information concerning fecal coliforms. These dehydrated, shelfstable plates are extremely lightweight and compact, which makes them inexpensive to transport. Furthermore, Petrifilms bypass many conventional monitoring expenses, such as petri dishes, perishable media, and sterilization equipment. Requiring only an incubator, such as egg incubator, Petrifilms present an advantage over traditional methods, by allowing the possibility for water authorities to analyze monitoring results on site. Currently, in Kumbo, samples are collected and shipped $400 \mathrm{~km}$ to a satellite facility for analysis. The
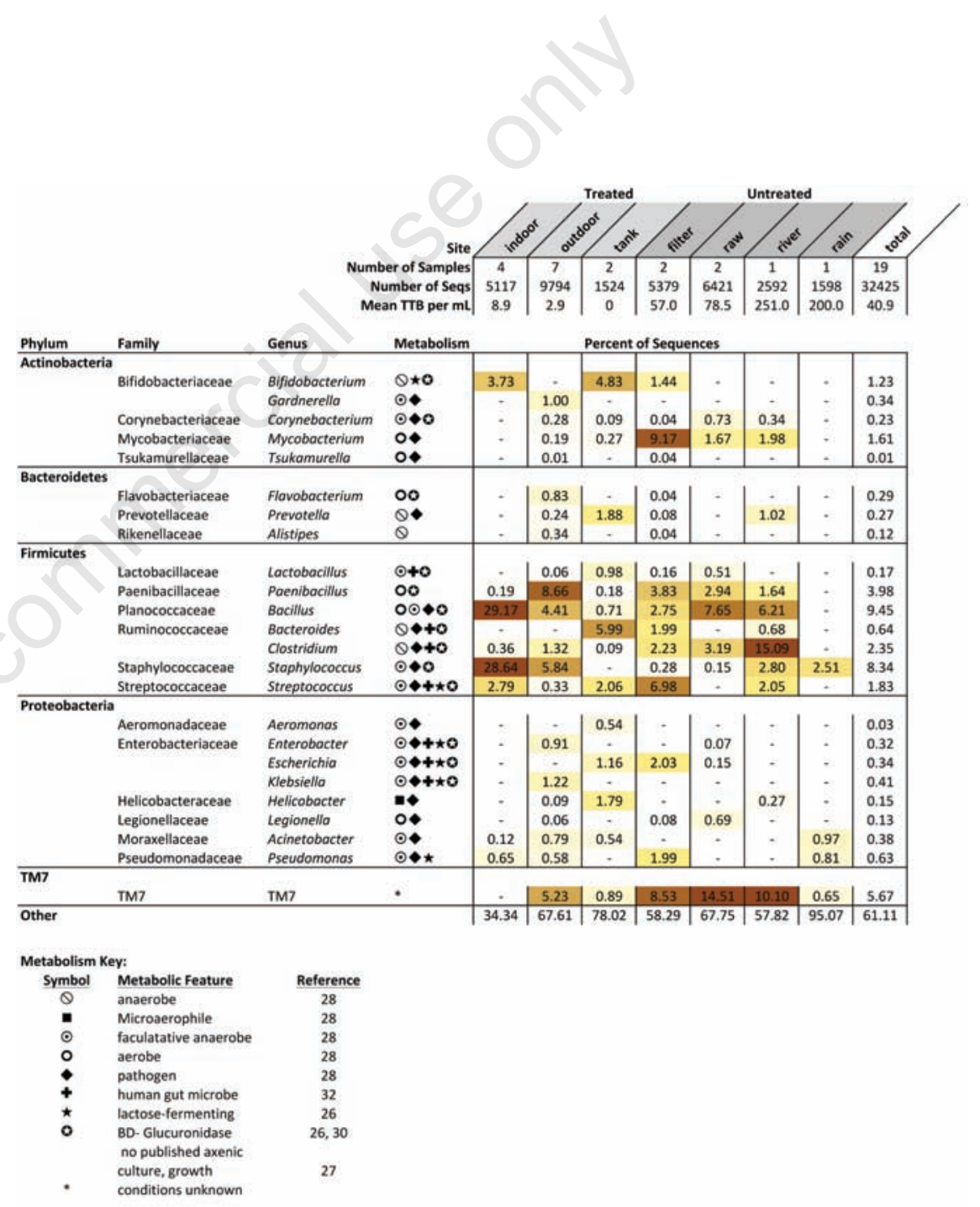

Figure 3. The heat map summarizes the distribution of bacterial taxa of known humanhealth interest or indicators of fecal contamination detected by phylogenetic analysis of pyrosequencing reads, clustered into genus-level groups. Values in the table represent percent abundances of particular taxa for each sample type (columns sum to $100 \%$ ), and are color coded on a gradient from white $(0 \%)$ to dark orange $(>10 \%)$ to highlight the abundant groups. ${ }^{*}$ Pathogen or Opportunistic Pathogen known in genera. 
KWA therefore has little control for how quickly the samples processed and pay approximately 600 USD per sampling event. Thus Petrifilms provide the opportunity for the KWA to expand its sampling frequency and improve time-to-results. To address the utility of using Petrifilms for water quality monitoring, we compared water collected on two occasions from various stages of water treatment and distribution. Results indicated that Petrifilm were reproducible between replicates and sampling dates. Additionally, cultivation on Petrifilms suggests that treatment by the KWA greatly improves water quality as compared with alternative, untreated river and rainwater sources. Culture data indicated that as untreated water for the KWA facility underwent sand filtration, the number of viable TTB and LFB decreased (Figure 2). However, the number of BDGB remained unchanged, suggesting that culturable $E$. coli was unaffected by sand filtration. When chlorinated water was collected from a protected tap at the water treatment facility, virtually no TTB, LFB, or BDGB were detected, suggesting that chlorination reduced viable coliforms to the WHO standard of zero cells per mL. ${ }^{10}$ As water was distributed, total TTB and LFB increased, most likely due to line infiltration, biofilm formation, and/or faucet contamination. Indoor and outdoor faucet samples had similar colony counts. Interestingly, a single restaurant water tap had higher levels of viable colonies than other indoor taps (29 vs 3 colonies per $\mathrm{mL}$ ), explaining the large standard deviation for indoor TTB. Restaurants handle many animal and food products, which may be contaminated with coliforms. The restaurant sample exemplifies the utility of the Petrifilm plates to detect poor sanitary conditions of possible public health concern.

Culture results suggest that alternative water sources, specifically river water and rainwater, were probably not as safe as KWA treated water. Although the city water did not meet WHO standards for purity, the number of viable coliforms in tap water was significantly lower in comparison to alternative water sources (Figure 2B; 4 vs 113 colonies per mL, $\left.\mathrm{P}=7.8 \times 10^{-8}\right)$. Culture results indicated that the River Bui was heavily contaminated with TTB, LFB, and BDGB and, therefore, unfit for human consumption. Furthermore, sequence data identified several potential pathogenic genera such as Clostridium, Mycobacterium, and Helicobacter in River Bui samples. In addition to surface water, the collection of rainwater for drinking is commonplace, though it often occurs into microbe-contaminated containers. Culture results showed that rainwater collected from a roof into unclean buckets was not safe; however, when the bucket was bleached and moved away from the roof, rainwater quality approached that of city treated water. Although we tested two common alternative water sources, rainwater and river water, the effectiveness of point-of-use treatment (e.g. iodine tablets) was not evaluated, as this practice is uncommon in Kumbo.

The $>32,000$ pyrosequences generated from filtered water samples, contained a rich diversity of bacterial types. Most of these were common water and soil organisms not typically associated with human disease. A minority, however, were aerobic and anaerobic bacteria known to exist in fecal material and/or cause illness. Despite the diversity of organisms detected, DNA sequencing has several important limitations pertinent to this study. First, the organisms detected may not be viable; DNA may be extracted from dead cells. Second, the short region of the $16 \mathrm{~S}$ gene amplified in this study was insufficient to classify beyond the genus-level of taxonomy for most sequences. Thus, the detection of genera that contain large numbers of environmental and humanassociated species, such as Bacillus, could not be further resolved. Furthermore, Escherichia and Shigella are not distinguishable by $16 \mathrm{~S}$ rRNA phylogenetic analysis. However despite these limitations, DNA sequencing provides significant benefits, including detection of difficult-to-culture organisms (e.g. anaerobes, TM7) and accurate identification based on genetics rather than phenotype. Indeed, sequencing identified many groups of anaerobic bacteria that are associated with fecal contamination, yet were not expected to be detected by Petrifilm cultivation.

Culture data may either under represent or misrepresent the diversity of species in an environment. ${ }^{28}$ Therefore, it is not surprising that weak correlations were found between $E$. coliShigella identified by sequencing and those nominally identified on culture plates by phenotype. In fact, only 14 E. coliShigella sequences were identified, all from sand-filtered water. In contrast, the culture plates detected nominal $E$. coli isolates in the river, raw and sand-filtered water samples with a total of 23 identifications (blue gas-forming colonies). Sequencing is generally considered to be more sensitive than culture; therefore we were surprised to see so few E. coliShigella sequences. It is likely that the culture plates do not only identify $E$. coli, but also other bacteria capable of $\beta$-D-glucuronidase and lactose-fermentative activity, such as some members of the genus Staphylococcus. ${ }^{29}$ False-positive $E$. coli identifications in the culture set may have arisen from closely related species in the Enterobacteriaceae family or other families that contain species with $\beta$-D-glucuronidase activity. ${ }^{29-31}$

Although Petrifilms are used routinely in the food industry, the volume of sample inoculated on the plate $(1 \mathrm{~mL})$ is smaller than U.S. EPA and WHO standardized tests.
Nevertheless, validation studies suggest that results obtained using these shelf-stable plates are not only reproducible, but compare favorably with more conventional fecal coliform tests. ${ }^{14,32,33} \mathrm{As}$ with any microbiological phenotypic test, in particular those that assay small volumes, false-negatives are a concern. A recent study demonstrated a false-negative rate of $8 \%$ for Petrifilms, which is considerably better than the $19 \%$ false-negative rate for Colilert ${ }^{\circledR}$, another inexpensive coliform test. ${ }^{34}$ In addition to having a lower false-negative rate, Petrifilms provide more visual data than a presence/absence test, such as Colilert ${ }^{\circledR}$, as colonies can be phenotypically distinguished by $\beta$-D-glucuronidase activity, gas-formation, and/or thermotolerance. Our data indicate that the occurrence of false-positives for nominal $E$. coli was more likely than false-negatives. $\beta$-Dglucuronidase activity in culture did, however, correlate with total sequence counts $(\mathrm{P}=0.0001)$ and apparent water quality, in that raw, untreated water had higher levels of $\beta$-Dglucuronidase bacteria than did chlorinated tap water. Therefore, even potential false positive results for $E$. coli detection may provide a useful index of water quality, as chlorination should inhibit all $\beta$-D-glucuronidase positive bacteria regardless of species origin.

As part of this study, we trained KWA technicians in the use of the Petrifilm plates. The technicians quickly learned sterile technique and proper plating, suggesting that other technicians throughout the developing world could easily be trained to use such a technology. The visual and colorimetric nature of the plates simplified interpretation of results without a need for special equipment. Furthermore, the directors of the KWA were very interested in the water quality monitoring because the plates were significantly less expensive, and likely more effective, than the current monitoring regimen. At less than 1 USD per sample, the agency could expand the frequency and number of sites sampled and therefore facilitate making more informed decisions concerning water treatment procedures.

\section{Conclusions}

The purpose of this study was to address two questions: (i) are shelf-stable $E$. coli/coliform count plates appropriate for routine monitoring of municipal water facilities in developing nations and (ii) is the Kumbo Water Authority piped-water system effective at providing clean drinking water? Our results demonstrate that shelf-stable, coliform-selective culture tests, such as 3M Petrifilms, are an appropriate technology for water quality monitoring in developing nations. The technology is reliable, easy to use with minimal training, compact for trans- 
port, and inexpensive. Furthermore, the results indicate that the Kumbo Water Authority offers improved water quality over alternative water sources such as rainwater and surface water, although the city did not meet WHO and western standards for microbial safety during our study period. By employing shelf-stable culture-based techniques in urban areas of developing nations, water authorities can monitor water quality more frequently. Increased monitoring data will allow authorities to determine the efficacy of treatment, increase their accountability to consumers, and ultimately assist in the reduction of water-borne diseases in the developing world.

\section{References}

1. World Health Organization. WHO - The global burden of disease: 2004 update [Internet]. Geneva: World Health Organization; 2008. http//www.who. int/healthinfo/global_burden_disease/2004_report_update/en/index.ht ml Accessed: 2011 July 25.

2. Jaglin $\mathrm{S}$. The right to water versus cost recovery: participation, urban water supply and the poor in sub-Saharan Africa. Environ Urban 2002;14:231-45.

3. World Health Organization/UNICEF. WHO Progress on sanitation and drinking-water 2010 update [Internet]. Geneva: World Health Organization; 2010. http//www.who.int/water_sanitation_health/p ublications/9789241563956/en/index.html Accessed: 2011 July 25.

4. Bureau Central des Recensements et Études de Population (BUCREP). Rapport de Présentation des Résultats Définitifs, 3e Recensement Général de la Population et de l'Habitat du Cameroun. Yaoundé: BUCREP; 2010.

5. Page B. Communities as the agents of commodification: the Kumbo Water Authority in Northwest Cameroon. Geoforum 2003;34:48398.

6. Verdzeka, E. Kumbo Water Authority announces measures to step up water supply [Internet]. L'Effort Camerounais. 2011 November

04. http//www.leffortcamerounais.com/2006/04/k umbo_suffers_f.html Accessed: 2012 March 3.

7. Shiyntum JN. Annual Management Report for the 2008 Fiscal Year. Kumbo: Kumbo Water Authority; 2009.

8. U.S. Environmental Protection Agency. Total coliform rule: a quick reference guide [Internet]. $\quad$ Epa.gov; 2010. http:/www.epa.gov/ogwdw/disinfection/tcr/pdf s/qrg_tcr_v10.pdf Accessed: 2011 Nov 14.

9. Daley B, Levenson M. Workers repairing water pipe; Boil order still in effect for 2 million
[Internet]. Boston.com. 2010 May 2. http:/www.boston.com/news/local/massachusetts/articles/2010/05/02/water_main_break/ Accessed: 2011 July 26.

10. World Health Organization. WHO - Guidelines for drinking-water quality, fourth edition [Internet]. Geneva: World Health Organization; 2011. http:/www.who.int/water_sanitation_health/p ublications/2011/dwq_guidelines/en/index.ht ml Accessed: 2011 July 25.

11. Stevens M, Ashbolt N, Cunliff D. Recommendations to change the use of coliforms as microbial indicators of drinking water quality. Australian Government: National Health and Medical Research Council; 2003. p 43.

12. Mattelet C. Household ceramic water filter evaluation using three simple low-cost methods: membrane filtration, 3M Petrifilm and hydrogen sulfide bacteria in northern region, Ghana [Master's Thesis]. Boston, MA: Massachusetts Institute of Technology; 2006. p 106.

13. Pearson AL, Roberts MC, Soge 00, et al. Utility of EC 3MTM petrifilmsTM and sanitary surveys for source water assessment in southwestern Uganda. Water SA 2008;34:279-283.

14. Vail JH, Morgan R, Merino CR, et al. Enumeration of waterborne Escherichia coli with petrifilm plates: comparison to standard methods. J Environ Qual 2003;32:368-73.

15. UltraClean® Fecal DNA Isolation Kit (12811) [Internet]. Carlsbad, CA, USA: MO Bio Laboratories, Inc. 1993-2012. http:/www. mobio.com/images/custom/file/protocol/12811.pdf Accessed: 2012 March 3.

16. NovaTaqTM DNA Polymerase and Kits (User Protocol TB309) [Internet]. Darmstadt, Germany: Merck KGaA. http//www.emdchemicals.com/chemdat/en_CA/Merck-USSite/USD/ ViewProductDocuments-File;sid =9ZQcvaoOibNcveVcfMlw6gLOchc0TcCpzbo C 6 x m j T W 8 q 5 y S A 2 X R_F 6 ww 4 s RAIlgy_oMoanXCKwluiPHVxDth-x-1GAFWbexg6knBIXluehmbRYRjkL5iez?ProductSK $\mathrm{U}=\mathrm{E}$ M D_B I 0 - 71007 \& D o c u m e n t Type=USP\&DocumentId=\%2Femd $\% 2 F b i o s c i$ ences\%2Fuserprotocols\%2Fen-US\%2FTB 309.pdf\&DocumentSource=GDS Accessed: 2012 March 3.

17. Frank JA, Reich CI, Sharma S, et al. Critical evaluation of two primers commonly used for amplification of bacterial 16S rRNA genes. Appl Environ Microbiol 2008;74:2461-70.

18. Lane D. 16S/23S DNA Sequencing. In: Nucleic acid techniques in bacterial systematics. New York, NY: John Wiley and Sons; 1991. pp 11575.

19. Frank DN. BARCRAWL and BARTAB: software tools for the design and implementation of barcoded primers for highly multiplexed DNA sequencing. BMC Bioinformatics 2009;10:362. 20. Nawrocki EP, Kolbe DL, Eddy SR. Infernal 1.0: inference of RNA alignments. Bioinformatics 2009;25:1335-7.

21. Cannone JJ, Subramanian S, Schnare MN, et al. The comparative RNA web (CRW) site: an online database of comparative sequence and structure information for ribosomal, intron, and other RNAs. BMC Bioinformatics 2002;3:2.

22. Hardesty B, Kramer G. Structure, function, and genetics of ribosomes. New York, NY: Springer-Verlag New York Inc.; 1986.

23. Haas BJ, Gevers D, Earl AM, et al. Chimeric 16S rRNA sequence formation and detection in Sanger and 454-pyrosequenced PCR amplicons. Genome Res 2011;21:494-504.

24. Wang Q, Garrity GM, Tiedje JM, Cole JR. Naïve Bayesian classifier for rapid assignment of rRNA sequences into the new bacterial taxonomy. Appl Environ Microbiol 2007;73:5261-7.

25. Frank D. XplorSeq: A software environment for integrated management and phylogenetic analysis of metagenomic sequence data. BMC Bioinformatics 2008;9:420.

26. Bergey's Manual of Determinative Bacteriology. 9th ed. Baltimore: Williams \& Wilkins; 1994.

27. Hugenholtz P, Tyson GW, Webb RI, et al. Investigation of candidate division TM7, a recently recognized major lineage of the domain bacteria with no known pure-culture representatives. Appl Environ Microbiol 2001;67:411-9.

28. Pace NR. A molecular view of microbial diversity and the biosphere. Science 1997;276:73440.

29. Manafi M, Kneifel W, Bascomb S. Fluorogenic and chromogenic substrates used in bacterial diagnostics. Microbiol Mol Biol Rev 1991;55:335-48.

30. Gloux K, Berteau 0, El Oumami H, et al. A metagenomic $\beta$-glucuronidase uncovers a core adaptive function of the human intestinal microbiome. Proc Natl Acad Sci U S A 2011;108 Suppl 1:4539-46.

31. Wu PC. Topics in Pathology for Hong Kong. Hong Kong: Hong Kong University Press; 1995.

32. Beloti V, De Souza JA, Barros MDF, et al. Evaluation of Petrifilm (TM) EC and HS for total coliforms and Escherichia coli enumeration in water. Braz J Microbiol 2003;34:301-4.

33. Schraft H, Watterworth LA. Enumeration of heterotrophs, fecal coliforms and Escherichia coli in water: comparison of $3 \mathrm{M}(\mathrm{TM})$ Petrifilm(TM) plates with standard plating procedures. J Microbiol Methods 2005;60:33542.

34. Chuang P, Trottier S, Murcott S. Comparison and verification of four field-based microbiological tests: H2S test, Easygel ${ }^{\circledR}$, Colilert ${ }^{\circledR}$, PetrifilmTM. J Water Sanitation Hyg for Dev 2011;01:68-85. 